\title{
Neonatologist performed echocardiography_hype, hope or nope
}

\author{
Luc Mertens ${ }^{1}$
}

Received: 8 August 2015 / Accepted: 14 August 2015 /Published online: 2 September 2015

(C) Springer-Verlag Berlin Heidelberg 2015

Ultrasound technology is evolving quickly, and miniaturization of technology has resulted in the development of handheld devices and compact ultrasound machines that can easily be used at the bedside. This has resulted in more widespread use of diagnostic ultrasound by different users. Point-of-care ultrasound is considered an extension of clinical examination and utilizes ultrasound technology to address specific clinical questions [11]. Focused cardiac assessment by echocardiography has been introduced into clinical practice in the emergency room, intensive care units and more recently also in the neonatal intensive care units (NICU). Neonatologists have embraced echocardiography for the hemodynamic assessment of neonates and preterm babies $[1,2,7,8]$. Specific indications include assessment of ductal patency, ventricular function, filling status for fluid management and pulmonary hypertension. This is a rapidly evolving field and an increasing number of neonatologists acquire echocardiographic skills to apply in the management of critically ill infants.

Successful introduction of any new imaging technique is critically dependent on the technical and interpretation skills of the providers, which require appropriate training. To address this specific training guidelines for neonatologists performing echocardiography have been proposed by different professional organizations worldwide. Two different approaches can be distinguished. A minimalistic training approach was introduced by neonatology groups in Australia

Communicated by Patrick Van Reempts

Luc Mertens

luc.mertens@sickkids.ca

1 The Labatt Family Heart Center, Cardiology, The Hospital for Sick Children, University of Toronto, 555 University Avenue, Toronto, ON M5G 1X8, Canada and New Zealand, which generally consists of attendance at a 2-day neonatal echocardiography course followed by performing a minimal number of 25 to 50 ultrasound examinations including imaging of different organ systems [1,2]. The maximalist approach is supported by North American and European professional organizations and includes echocardiographic training of neonatologists in pediatric echocardiography laboratories followed by more specific training in a NICU environment [9]. The reasoning behind the minimalistic approach is that the scope of practice is very limited. This implies you do not need extensive training to adequately utilize the technique in clinical practice. The maximalist approach focuses on the safety of practice and takes into consideration that around $0.5-1 \%$ of all newborn infants have congenital heart disease, and misdiagnosing critical structural heart disease could result in errors in clinical management. Based on this, neonatologists should be able to identify critical cardiac structural abnormalities. Alternatively, a pediatric cardiologist or neonatologist with training in structural heart disease evaluates every first study in a critically ill newborn. The paper by Singh et al. published in the current issue of the European Journal of Pediatrics should be interpreted in this context. It is a consensus document on neonatologist-performed echocardiography training and accreditation generated by a group of neonatologists and cardiologists in the UK. This document largely follows the more combined North AmericanEuropean approach and recommends training neonatologists in pediatric echocardiography laboratories. The proposed program allows training neonatologists and pediatricians up to a level where they should be able to reliably identify congenital heart disease in newborns. This is particularly useful in NICUs where access to pediatric cardiology is not readily available.

Related to the training, some of the controversy could be brought down to the question of the actual scope and utility of neonatologist-performed echocardiography in patient 
management in the NICU. A recent study from Calgary retrospectively looked at the therapeutic impact of targeted neonatal echocardiography on clinical management [4]. Of the 303 studies performed, $126(41.5 \%)$ resulted in changes in clinical management, the majority related to treatment of ductal patency $(45 \%)$. Other changes involved adjustment of inotropic support or fluid administration (17.8\%) and repositioning of an umbilical venous catheter (5\%). Interestingly, one patient with unsuspected transposition of the great arteries was detected, demonstrating the importance of the ability to diagnose congenital heart disease. The set-up in Calgary involved collaboration with a pediatric cardiologist evaluating every first study performed by a neonatologist. This indicates that knowledge on structural heart disease is indeed required. A concern remains that while the study demonstrates that clinicians based some of the clinical decisions on echocardiographic data, it does not show that these management changes actually influenced outcomes.

For definition of training requirements, the impact of the scope of neonatologist-performed echocardiography on the skill set required to perform the actual examinations needs to be considered. Assessing ductal patency requires understanding of aortic arch anatomy and identification of ductdependent cardiac abnormalities. To evaluate the hemodynamic impact of a patent duct, different measurements (chamber sizes, mitral inflow, shunt directionality, etc.) have to be acquired and interpreted. As cardiac structures are small in preterm infants, small measurement errors have a more significant impact on interpretation of the data. Targeted neonatal echocardiography is used to assess volume status in preterm infants. This is a challenging question as even in adults, echocardiographic assessment of filling status and diastolic function is difficult and requires extensive training and understanding of different parameters influencing ventricular filling and determining preload [10]. In preterm infants, there is very limited knowledge on diastolic ventricular properties and before generalized utilization of echocardiographic techniques to guide fluid management, research is required to define which echocardiographic parameters can be used to evaluate fluid status. It should not be based on subjective assessment. Also, quantitative assessment of systolic function remains challenging in preterm infants. The neonatal heart is characterized by limited contractile and diastolic reserve, is more sensitive to loading conditions, and the small size of the heart makes the measurements more difficult and variable. Quantitative parameters are available, but the influence of the transitional changes on the different cardiac parameters is still being explored in research studies [6]. Criteria for how certain measurements influence decision-making have not been established and would require more extensive research. Validation studies of cardiac output measurements as performed by comparing echocardiographic measurements with flow measurements obtained by cardiac magnetic resonance imaging are important for further development of the technique [3]. Neonatologist-performed echocardiography is also used in the assessment of pulmonary hypertension of the newborn. This always requires first ruling out congenital heart defects causing pulmonary hypertension (total anomalous pulmonary venous drainage or large shunts). Assessment of pulmonary hypertension also requires assessment of right ventricular (RV) function. In infants, markers for RV dysfunction and criteria for how to define severity of RV dysfunction are still poorly defined and research in this field is still ongoing [5]. Finally, the skills required for guiding line placement and evaluation of pericardial effusions are probably more limited and really consist excellent point-of-care indications with clear benefits.

It is remarkable that NICUs have introduced TNE without the necessary clinical validation and limited definition of the criteria that can be used for changing clinical management. Before investing in expensive machines and training a significant number of neonatologists, data on how this affects clinical management and most importantly clinical outcomes for the NICU patients need to be collected. If echocardiography is to be used for clinical decision-making in critically ill children, the techniques need to be further validated, normal values have to be defined and most importantly, criteria for guiding therapy need to be established. There is excellent ongoing clinical research in the field, and different groups are moving forward with better understanding of neonatal hemodynamics. While waiting for these clinical studies, clinical utilization of TNE should be limited to those with more extensive training as defined UK training.

\section{References}

1. Evans N (2000) Echocardiography on neonatal intensive care units in Australia and New Zealand. J Paediatr Child Health 36(2):169171

2. Evans N, Gournay V, Cabanas F, Kluckow M, Leone T, Groves A et al (2011) Point-of-care ultrasound in the neonatal intensive care unit: international perspectives. Semin Fetal Neonatal Med 16(1): 61-68

3. Ficial B, Finnemore AE, Cox DJ, Broadhouse KM, Price AN, Durighel G et al (2013) Validation study of the accuracy of echocardiographic measurements of systemic blood flow volume in newborn infants. J Am Soc Echocardiogr 26(12):1365-1371

4. Harabor A, Soraisham AS (2015) Utility of targeted neonatal echocardiography in the management of neonatal illness. J Ultrasound Med 34(7):1259-1263

5. Jain A, Mohamed A, El-Khuffash A, Connelly KA, Dallaire F, Jankov RP et al (2014) A comprehensive echocardiographic protocol for assessing neonatal right ventricular dimensions and function in the transitional period: normative data and z scores. J Am Soc Echocardiogr 27(12):1293-1304

6. James AT, Corcoran JD, Jain A, McNamara PJ, Mertens L, Franklin $O$ et al (2014) Assessment of myocardial performance in preterm infants less than 29 weeks gestation during the transitional period. Early Hum Dev 90(12):829-835 
7. Kluckow M, Seri I, Evans N (2007) Functional echocardiography: an emerging clinical tool for the neonatologist. J Pediatr 150(2): 125-130

8. Kluckow M, Seri I, Evans N (2008) Echocardiography and the neonatologist. Pediatr Cardiol 29(6):1043-1047

9. Mertens L, Seri I, Marek J, Arlettaz R, Barker P, McNamara P et al (2011) Targeted neonatal echocardiography in the neonatal intensive care unit: practice guidelines and recommendations for training. Writing Group of the American Society of Echocardiography (ASE) in collaboration with the European Association of
Echocardiography (EAE) and the Association for European Pediatric Cardiologists (AEPC). J Am Soc Echocardiogr 24(10): $1057-1078$

10. Nagueh SF, Appleton CP, Gillebert TC, Marino PN, Oh JK, Smiseth OA et al (2009) Recommendations for the evaluation of left ventricular diastolic function by echocardiography. Eur J Echocardiogr 10(2):165-193

11. Solomon SD, Saldana F (2014) Point-of-care ultrasound in medical education — stop listening and look. N Engl J Med 370(12):10831085 